\title{
PHARMACOGENETICS IN ONCOLOGY
}

\author{
Danesi R, Di Paolo A, Bocci G, *Crea F, Del Tacca M
}

Division of Pharmacology, Department of Internal Medicine, University of Pisa, Via Roma 55, 56126 Pisa (Italy)

*Sant'Anna School of Advanced Studies, Piazza Martiri della Libertà, 33 - 56127 Pisa (Italy)

\section{Address for correspondence:}

Romano Danesi, MD, PhD

Division of Pharmacology and Chemotherapy

Department of Internal Medicine

55, Via Roma 56126 Pisa, Italy

E-mail: r.danesi@med.unipi.it

Phone: +39-050-992632

Fax: +39-050-562020

Running title: Pharmacogenetics in oncology

Conflict of interest statement: The Authors declare that no conflict of interest arises in relation to the present article.

Role of the funding source: The Authors had no funding sources for this article. 


\begin{abstract}
The number of studies reporting a relationship between DNA sequence variants and cancer treatment outcome are increasing in number. In particular, the following associations were found: dihydropyrimidine dehydrogenase (DPD) gene mutations and severe 5-FU toxicity, epidermal growth factor receptor mutations (EGFR) and responsiveness of NSCLC to gefitinib, ERCC1 polymorphisms and activity of cisplatin, genetic variants of UGT1A1 gene and severe neutropenia by irinotecan, thymidylate synthase (TS) gene polymorphisms and 5-FU sensitivity, and cytidine deaminase (CDA) genotype and expression of equilibrative nucleoside transporter-1 (hENT1) and response to gemcitabine. The next step in pharmacogenetic research should be the validation of these findings in randomized prospective trials, specifically designed to compare the outcome of treatment selected on the basis of patient's genotype vs. standard approach. In conclusion, the improvement in genotyping technologies, combined with efficient and cost-effective analytical methods, may fulfill the promise of personalizing the treatment offered to cancer patients.
\end{abstract}

Keywords: Pharmacogenetics, cancer, chemotherapy, germline, somatic, polymorphisms 


\section{Introduction}

Cancer is the result of a multistep process of mutations in key regulatory genes and epigenetic alterations that result in loss of balanced gene expression. Dominant oncogenes contribute to malignant transformation by promoting uncontrolled cell proliferation, blocking normal differentiation and preventing apoptosis, whereas recessive tumour suppressor genes encode proteins that negatively regulate cell proliferation. Within the past two decades, the molecular pathology of cancer has achieved a wider recognition and now encompasses a rapidly expanding field that discovers novel targets for drug development. Cancer chemotherapy has progressed since its introduction into clinical practice and represents the most promising treatment modality. Its use, however, is limited by the inability to predict the response of the tumor, and the choice of treatment protocols is still mostly empirical. This approach has important limitations because it does not take account of tumor (somatic) and host (germline) genetic variability and it is not surprising that patients with apparently identical tumors do not always respond to the same drugs or have the same severity of adverse reactions (1).

The individualization of therapy is the longstanding goal of pharmacologists; since the 1950s, in vitro tests have been developed that identify effective drugs and avoid unnecessary toxicity (1). With the completion of the Human Genome Project and identification of genes implicated in the development of cancer, the next tasks were to understand the influence of oncogenes and tumor suppressor genes on drug susceptibility and apply genetic profiling to the choice of pharmacological treatment. The influence of the genetic background on responses to anti-cancer agents is particularly interesting considering that the reasons for the success of chemotherapy in some cancers (i.e. testicular seminoma) and the discouraging results in others (i.e. exocrine pancreas cancer) are still unclear. In this context, pharmacogenetics seeks to identify genetic factors that contribute to interpatient and interdrug variation in responses to anti-neoplastic chemotherapy (1). 
Polymorphisms in genes coding for metabolizing enzymes and drug transporters can affect drug efficacy and toxicity (2). Pharmacogenomics aims to identify individuals predisposed to high risk of toxicity and low response from standard doses of anticancer drugs. The present article focuses on the clinical significance of polymorphisms in drug-metabolizing enzymes and drug transporters in influencing efficacy and toxicity of anticancer therapy. The most important examples to demonstrate the influence of pharmacogenetics on anticancer therapy are dihydropyrimidine dehydrogenase (DPD) gene mutations and severe 5-fluorouracil (5-FU) toxicity, epidermal growth factor receptor mutations (EGFR) and responsiveness of non-small cell lung cancer (NSCLC) to gefitinib, ERCC1 polymorphisms and activity of cisplatin, genetic variants of UDP glucuronosyltransferase 1A1 (UGT1A1) gene and severe neutropenia by irinotecan, thymidylate synthase (TS) gene polymorphisms and 5-FU sensitivity, and cytidine deaminase (CDA) genotype and expression of equilibrative nucleoside transporter-1 (hENT-1) and response to gemcitabine. However, in most other anticancer therapies no clear association has been found for polymorphisms in drug-metabolizing enzymes and drug transporters and pharmacokinetics or pharmacodynamics of anticancer drugs. Evaluation of different regimens and tumor types showed that polymorphisms can have different, sometimes even contradictory, pharmacokinetic and pharmacodynamic effects in different tumors in response to different drugs (2). The clinical application of pharmacogenetics in cancer treatment therefore requires more detailed information regarding the different polymorphisms in drug-metabolizing enzymes and drug transporters. A greater understanding of complexities in pharmacogenetics is needed before individualized therapy can be applied on a routine basis (2). This review focuses on the influence of selected genes on the activity of anticancer agents: examples described illustrate the range of responses and toxicities that result from the interaction of drugs with genes expressed within tumor and non-tumor cells, highlight how genetics will impact on pharmacology, and underscore the role of pharmacogenetics in making therapeutic decisions and optimizing treatment. 


\section{DPD gene mutations and severe 5-FU toxicity}

The identification of genetic factors associated with either responsiveness or resistance to 5-FU chemotherapy, as well as genetic factors predisposing patients to the development of severe 5-FUassociated toxicity, is increasingly being recognised as an important field of study. DPD plays a key role in the catabolism of 5-FU to 5-fluoro-5,6-dihydrouracil (5-FDHU), and as such, an impairment of DPD has been recognized as an important factor for altered 5-FU and 5-FDHU pharmacokinetics, predisposing patients to the development of severe 5-FU-associated toxicity (3). Patients with DPD deficiency have an increased risk of developing grade IV neutropenia. In addition, the onset of toxicity occurred twice as fast compared with patients with a normal DPD activity. To date, 39 different mutations and polymorphisms have been identified in DPYD. The IVS14+1G $>$ A mutation proved to be the most common one and was detected in $24-28 \%$ of all patients suffering from severe 5-FU toxicity. Thus, a deficiency of DPD appears to be an important pharmacogenetic syndrome (3). In order to identify patients at risk of toxicity by 5-FU, several methods have been proposed, including germline genotypization (4) and phenotyping through administration of 5-FU test-dose (5). In a recent study the pharmacokinetics of 5-FU/5-FDHU and DPD activity in peripheral blood mononuclear cells (PBMCs) were examined in gastrointestinal cancer patients given a test dose of 5-FU, $250 \mathrm{mg} / \mathrm{m}^{2}$ before the planned 5 -FU treatment of 370 $\mathrm{mg} / \mathrm{m}^{2}$ plus L-folinic acid, $100 \mathrm{mg} / \mathrm{m}^{2}$. The 5-FU test dose was well tolerated in all patients; in 3 of them marked alterations of 5-FU/5-FDHU pharmacokinetics (i.e., increased 5-FU half-life and reduced 5-FU total body clearance) were observed; they were excluded from 5-FU treatments and given irinotecan, which was well tolerated. A significant correlation between 5-FU AUC and 5FDHU AUC was found, whereas a weaker correlation between PBMC DPD activity and both 5FDHU AUC and 5-FDHU Cmax was observed. No relationships between PBMC DPD activity and 5-FU toxicities were found, whereas 5-FDHU pharmacokinetics was associated with the risk of moderate to severe neutropenia and diarrhea (5). 
Indeed, 5-FU and 5-FDHU pharmacokinetics is an important parameter to predict response to adjuvant chemotherapy in radically-resected colorectal cancer patients. Individual plasma pharmacokinetic parameters of 5-FU and 5-FDHU were determined at the first cycle and correlated with disease-free survival. The main finding was a lower area under the time/concentration curve (AUC) of 5-FU in patients who recurred with respect to other patients. Furthermore, AUC values lower than $8.4 \mathrm{~h} \mathrm{x} \mathrm{mg/L} \mathrm{together} \mathrm{with} \mathrm{lymph} \mathrm{node} \mathrm{involvement} \mathrm{and} \mathrm{the} \mathrm{interruption} \mathrm{of} \mathrm{treatment} \mathrm{or}$ reduction of doses were identified as risk factors at univariate analysis, thus suggesting that pharmacokinetics of 5-FU is an important factor for predicting disease recurrence in colorectal cancers (6).

\section{EGFR mutations and responsiveness of NSCLC to gefitinib}

The disappointing results of long-term survival among patients with NSCLC may reflect the lack of knowledge of the ways in which molecular abnormalities of neoplastic cells affect responsiveness to anticancer therapy. Remarkable advances in the understanding of NSCLC cancer biology have been made over the past decade, including the discovery of critical mutations in oncogenes (i.e., mutation of K-Ras and c-myc gene), as well as the loss of tumor-suppressor genes, such as TP53, p16INK4, or Rb. The future challenge of NSCLC chemotherapy relies on the identification of molecular markers that are predictive of drug sensitivity and help in the selection of chemotherapeutic agents best suited to the individual patient. Other intriguing issues will be the identification of the optimal drug sequence in combination regimens, as well as polymorphisms of genes involved in severe toxicities (7).

The EGFR family, including EGFR, HER2, HER3, and HER4, is implicated in the development and progression of cancer, and is expressed in many human epithelial malignancies, including NSCLC. Several molecules were synthesized to inhibit the extracellular domain of EGFR, such as cetuximab, or the EGFR tyrosine kinase domain, such as gefitinib and erlotinib. Gefitinib and 
erlotinib produce objective response rates in about $10 \%$ of advanced NSCLC. Among clinical characteristics, female gender, adenocarcinoma histology, never smoking history, East Asian ethnicity are probably the most relevant factors. The presence of specific EGFR gene mutations or EGFR gene amplification confers a particularly sensitive phenotype (8).

The EGFR mutations are distributed throughout the kinase domain, but a deletion in exon 19 and the point mutation L858R in exon 21 account for approximately 90\%, which confer a greater response to gefitinib treatment, compared with other types of EGFR mutations. These EGFR mutations in the tyrosine kinase domain are somatic and involve a subset of lung cancers patients; the pathogenesis of these tumors does not involve the carcinogenic effect of smoke. In Japan, the EGFR mutations are detected in approximately $30 \%$ of overall NSCLC and approximately $40 \%$ of surgically resected adenocarcinomas. The morphological features of adenocarcinomas harboring the mutations were reported to be frequent in those with bronchioloalveolar features (9).

Finally, an important issue in EGFR target-specific therapy is the combination with standard cytotoxic agents. Patients with NSCLC stage IIIa/b pN2/3 were treated with at least three cycles of chemotherapy before undergoing surgery. EGFR expression was evaluated on mediastinal nodes at the time of initial diagnosis and on both the primary tumor and residual metastatic nodes after treatment (10). Chemotherapy was not associated with disappearance of EGFR immunoreactivity and in most patients the initial score was maintained. Moreover, in 6 patients with negative EGFR immunostaining before chemotherapy, 4 turned on the expression of EGFR from 0 to $2+/ 3+$, thus suggesting a very good agreement of EGFR status before and after chemotherapy in EGFR-positive NSCLC (10).

\section{ERCC1 polymorphisms and activity of cisplatin}

Cisplatin has a broad range of activity in malignant disease and is used to treat many types of cancer. It is particularly active against germ cell tumors and epithelial ovarian cancer and play a 
primary role in the treatment of SCLC and NSCLC, cervical cancer, head and neck cancer, colorectal cancer, and bladder cancer. Resistance to platinum-based chemotherapy can be intrinsic or acquired and may be mediated by factors outside or within the cancer cell or at its cell membrane (11). Resistance to platinum chemotherapy is multifactorial; there are DNA repair pathways that protect DNA from chemical injury: nucleotide excision repair (NER), mismatch repair (MMR), double-strand break repair (DSBR), base excision repair (BER), and direct repair. NER seems to play a key role in mediating resistance or sensitivity to platinum chemotherapeutic agents (11). NER is a highly conserved DNA repair pathway that repairs DNA lesions which alter the helical structure of the DNA molecule and interfere with DNA replication and transcription. Important steps in this pathway include the recognition of DNA damage and demarcation of the specific area affected, followed by the formation of a complex to unwind the damaged portion and excise it (11). Finally, the excised area is resynthesized and ligated to maintain the integrity of the DNA molecule. The excision repair cross-complementation group 1 (ERCC1) protein plays a key role in nucleotide excision repair. ERCC1 dimerizes with xeroderma pigmentosum complementation group F (XPF), and this complex is required for the excision of the damaged DNA. Evaluation of ERCC1 transcript levels in tumor samples taken from ovarian (12) and colorectal (13) tumor tissues had shown an inverse correlation with either response to platinum therapy or survival.

More recently, tumor samples from patients with metastatic lung cancer who participated in the International Adjuvant Lung Cancer trial were retrospectively evaluated by immunohistochemical analysis of ERCC1. This study showed a statistically significant survival benefit in patients with low levels of ERCC1 who had received platinum-based chemotherapy, compared to patients with low levels of ERCC1 who did not receive chemotherapy and patients with high levels of ERCC1 who received cisplatin chemotherapy (14). These data, however, were not confirmed by other studies (15). 


\section{Genetic variants of UGT1A1 gene and severe neutropenia by irinotecan}

The uridine diphosphate glucuronosyltransferase (UGT) 1A1 and 1A9 isoforms are involved in the phase II biotransformation of the irinotecan metabolite, SN-38 (16). An exceptionally large number of UGT1A and 2B variants have been described with altered functionality in vitro (17), one of the most common being UGT1A1*28 (17). A clinical association between the drug's active metabolite SN-38 and toxicity has been found and by performing UGT1A1 genetic testing, some studies have been able to predict which patients receiving irinotecan will experience the toxicity (reviewed by Perera et al. [18]). The functional consequence of the UGT1A1 TATA box variants [A(TA)6TAA to A(TA)7TAA, UGT1A1*28], UGT1A9 766G>A (D256N; UGT1A9*5), and UGT1A9 98T>C (M33T; UGT1A9*3) genotypes was assessed in Caucasian patients treated with irinotecan (19). The pharmacogenetic/pharmacokinetic study was performed after the first course of irinotecan in patients with solid tumors. For UGT1A9*5, non-variant alleles were observed, whereas for UGT1A9*3, 1 patient with the variant allele was found (allele frequency, 0.633\%). The distribution of UGT1A1*28 showed 51.1\% wild-type patients (Wt), 43.1\% heterozygotes (Het), and 5.8\% homozygotes (Var). The median AUC ratio of SN-38G to $\mathrm{SN}-38$ was significantly reduced in carriers of the variant UGT1A1*28 allele. It is concluded that UGT1A9 functional variants are rare in Caucasians and likely to be clinically insignificant in irinotecan regimens. Screening for the UGT1A1*28 polymorphism may identify patients with altered SN-38 pharmacokinetics (19).

\section{TS gene polymorphisms and 5-FU sensitivity}

TS is an important target for 5-FU, 5-fluorodeoxyuridine (FUDR), UFT, S-1, and capecitabine. Overexpression of TS is linked to resistance to TS inhibitors. The TS gene promoter enhancer region (TSER) contains two different polymorphisms which can influence TS mRNA transcriptional and translational efficiency: a polymorphic tandem repeat sequence (2 or 3 repeats; 2R and 3R) and a single nucleotide polymorphism (SNP), G > C, within the second repeat of the 3R 
alleles. Polymorphic tandem repeats located in the TS enhancer region (TSER) have been shown to influence protein expression. Three copies (TSER*3) of the tandem repeat give a 2.6-fold greater in vitro TS levels than 2 copies (TSER*2) (20), while the effect of alleles containing 4 (TSER*4), 5 (TSER*5), and 9 (TSER*9) copies of the tandem repeat have unclear significance. Stage III colorectal cancer patients with the TSER*3/TSER*3 genotype do not enjoy the clinical benefit of adjuvant 5-FU observed in patients with the TSER*3/TSER*2 or TSER*2/TSER*2 genotype (20). This finding is clinically relevant because the frequency of TSER*3/TSER*3 genotype is $30 \%$ in Caucasian patients. Prospective clinical trials should be able to confirm the impact of TSER genotype on clinical outcome of patients treated with TS inhibitors (20).

A study on matched tumor and normal mucosa demonstrated no differences in TS mRNA levels between cancer and normal tissue. High-grade, undifferentiated tumors (WHO grade 3) had significantly higher mRNA levels of TS with respect to moderately differentiated (WHO grade 2) carcinomas. However, TS mRNA expression was significantly decreased in homozygous TSER*3G/3G with respect to pooled homozygous TSER*2/2 and heterozygous TSER*2/3 genotypes. Thus, TSER*3G/3G and high-grade histology are associated with significant variation in TS gene expression in tumour samples (21).

\section{CDA genotype and expression of hENT-1 and response to gemcitabine}

Gemcitabine (2',2'-difluorodeoxycytidine) is a deoxycytidine analogue with antineoplastic activity against solid tumors. Gemcitabine is transported into cells by the equilibrative and concentrative nucleoside transport systems (ENT and CNT), followed by 1) metabolic activation to form phosphate derivatives that are either incorporated into DNA or competitively inhibits ribonucleotide reductase, or 2) inactivation by deamination catalyzed by cytidine deaminase (CDA) (22).

A study characterized the expression pattern of genes involved in gemcitabine activity in pancreas cancer and correlated this finding with treatment outcome. The transcription analysis of human 
equilibrative nucleoside transporter-1 (hENT1), deoxycytidine kinase (dCK), 5'-nucleotidase (5'NT), cytidine deaminase (CDA), and ribonucleotide reductase subunits M1 and M2 (RRM1 and RRM2) was done by quantitative reverse transcription-PCR in tumor tissue isolated by laser microdissection from surgical or biopsy samples of patients. hENT1 expression was significantly correlated with clinical outcome. Patients with high levels of hENT1 had a significantly longer overall survival. Similar results were obtained with disease-free survival and time to disease progression, and the multivariate analysis confirmed the prognostic significance of hENT1 (23).

The analysis of CDA Lys27Gln polymorphism in blood samples of advanced NSCLC patients treated with cisplatin-gemcitabine combination demonstrated a significant association with improved clinical benefit, occurrence of severe neutropenia and thrombocytopenia, as well as longer time ti progression and overall survival in patients with CDA Lys27Lys genotype. These results may be explained by the higher systemic gemcitabine exposure associated with Lys27Lys CDA (24).

\section{Genetic variants of VEGF and VEGFR and clinical outcome of anti-angiogenic treatments}

The concept of targeting tumor angiogenesis has moved from a pioneering research field into clinical practice and the novel drugs now available are defining a new and promising avenue of targeted cancer therapy. As with many other target-specific treatments, however, the need for a rational selection of patients to be administered anti-angiogenic treatments is emerging, since the clinical activity of these agents appears to be limited to specific patients and is not predictable on the basis of standard approaches. Candidate targets for treatment optimization are VEGF and their VEGFR, and the activity of drugs inhibiting VEGF and VEGFR signal transduction pathways are likely to be influenced by their intrinsic biological activities and the regulation of the gene expression of VEGF and VEGFR as well (for review see 25). The pharmacogenetic approach to anti-angiogenic therapy should be considered a possible strategy for delivering the optimal 
treatment to specific groups of patients affected by tumors as well as by other pathological conditions dominated by pathological angiogenesis, including age-related macular degeneration or endometriosis. While pharmacogenetic studies are building stronger foundations for the systematic investigations of phenotype-genotype relationships in many fields of medicine, pharmacogenetic data regarding anti-angiogenic drugs are still lacking. Many genetic variants are being discovered and single nucleotide polymorphisms of VEGF and VEGFR genes appear to be able to affect VEGF transcription, affinity to its receptor and biological activity of signal transduction pathway.

A number of studies demonstrated a significant association between prostate (27), thyroid (28), bladder (29) and breast cancer (30) risk and VEGF polymorphisms but definitive data correlating VEGF genotypes with response to anti-angiogenic treatments are lacking. 


\section{References}

1. Danesi R, De Braud F, Fogli S, Di Paolo A, Del Tacca M. Pharmacogenetic determinants of anticancer drug activity and toxicity. Trends Pharmacol Sci 2001;22:420-6.

2. Bosch TM. Pharmacogenomics of drug-metabolizing enzymes and drug transporters in chemotherapy. Methods Mol Biol 2008;448:63-76.

3. Lee A, Ezzeldin H, Fourie J, Diasio R. Dihydropyrimidine dehydrogenase deficiency: impact of pharmacogenetics on 5-fluorouracil therapy. Clin Adv Hematol Oncol 2004;2:527-32.

4. Mercier C, Ciccolini J. Profiling dihydropyrimidine dehydrogenase deficiency in patients with cancer undergoing 5-fluorouracil/capecitabine therapy. Clin Colorectal Cancer 2006;6:288-96.

5. Bocci G, Barbara C, Vannozzi F, Di Paolo A, Melosi A, Barsanti G, Allegrini G, Falcone A, Del Tacca M, Danesi R. A pharmacokinetic-based test to prevent severe 5-fluorouracil toxicity. Clin Pharmacol Ther 2006;80:384-95.

6. Di Paolo A, Lencioni M, Amatori F, Di Donato S, Bocci G, Orlandini C, Lastella M, Federici F, Iannopollo M, Falcone A, Ricci S, Del Tacca M, Danesi R. 5-Fluorouracil pharmacokinetics predicts disease-free survival in patients administered adjuvant chemotherapy for colorectal cancer. Clin Cancer Res 2008;14:2749-55.

7) Danesi R, Pasqualetti G, Giovannetti E, Del Tacca M. The role of pharmacogenetics in adjuvant treatment of non-small cell lung cancer. $J$ Thorac Oncol 2007;2(5 Suppl):S27-30.

8. Metro G, Finocchiaro G, Toschi L, Bartolini S, Magrini E, Cancellieri A, Trisolini R, Castaldini L, Tallini G, Crino L, Cappuzzo F. Epidermal growth factor receptor (EGFR) targeted therapies in non-small cell lung cancer (NSCLC). Rev Recent Clin Trials 2006;1:1-13.

9. Yatabe Y, Mitsudomi T. Epidermal growth factor receptor mutations in lung cancers. Pathol Int 2007;57:233-44. 
10) De Pas T, Pelosi G, de Braud F, Veronesi G, Curigliano G, Leon ME, Danesi R, Noberasco C, d'Aiuto M, Catalano G, Viale G, Spaggiari L. Modulation of epidermal growth factor receptor status by chemotherapy in patients with locally advanced non-small-cell lung cancer is rare. $J$ Clin Oncol 2004;22:4966-70.

11. Martin LP, Hamilton TC, Schilder RJ. Platinum resistance: the role of DNA repair pathways. Clin Cancer Res 2008;14:1291-5.

12. Smith S, Su D, Rigault de la Longrais IA, Schwartz P, Puopolo M, Rutherford TJ, Mor G, Yu $\mathrm{H}$, Katsaros D. ERCC1 genotype and phenotype in epithelial ovarian cancer identify patients likely to benefit from paclitaxel treatment in addition to platinum-based therapy. $J$ Clin Oncol 2007;25:5172-9.

13. Martinez-Balibrea E, Abad A, Aranda E, Sastre J, Manzano JL, Díaz-Rubio E, Gómez-España A, Aparicio J, García T, Maestu I, Martínez-Cardús A, Ginés A, Guino E; on behalf of the Spanish Group for the Treatment of Digestive Tumours (TTD group). Pharmacogenetic approach for capecitabine or 5-fluorouracil selection to be combined with oxaliplatin as first-line chemotherapy in advanced colorectal cancer. Eur J Cancer 2008 (doi:10.1016/j.ejca.2008.03.025)

14. Olaussen KA, Dunant A, Fouret P, Brambilla E, André F, Haddad V, Taranchon E, Filipits M, Pirker R, Popper HH, Stahel R, Sabatier L, Pignon JP, Tursz T, Le Chevalier T, Soria JC; IALT Bio Investigators. DNA repair by ERCC1 in non-small-cell lung cancer and cisplatin-based adjuvant chemotherapy. N Engl J Med 2006;355:983-91.

15. Booton R, Ward T, Ashcroft L, Morris J, Heighway J, Thatcher N. ERCC1 mRNA expression is not associated with response and survival after platinum-based chemotherapy regimens in advanced non-small cell lung cancer. J Thorac Oncol 2007;2:902-6.

16) Nagar S, Remmel RP. Uridine diphosphoglucuronosyltransferase pharmacogenetics and cancer. Oncogene 2006;25:1659-72.

17. http://www.pharmacogenomics.pha.ulaval.ca/sgc/ugt_alleles (last accessed May 29th, 2008) 
18) Perera MA, Innocenti F, Ratain MJ. Pharmacogenetic testing for uridine diphosphate glucuronosyltransferase 1A1 polymorphisms: are we there yet? Pharmacotherapy 2008;28:755-68.

19. Paoluzzi L, Singh AS, Price DK, Danesi R, Mathijssen RH, Verweij J, Figg WD, Sparreboom A. Influence of genetic variants in UGT1A1 and UGT1A9 on the in vivo glucuronidation of SN-38. J Clin Pharmacol 2004;44:854-60.

20. Marsh S, McLeod HL. Thymidylate synthase pharmacogenetics in colorectal cancer. Clin Colorectal Cancer 2001;1:175-8.

21. Amatori F, Di Paolo A, Del Tacca M, Fontanini G, Vannozzi F, Boldrini L, Bocci G, Lastella M, Danesi R. Thymidylate synthase, dihydropyrimidine dehydrogenase and thymidine phosphorylase expression in colorectal cancer and normal mucosa in patients. Pharmacogenet Genomics 2006;16:809-16.

22. Gilbert JA, Salavaggione OE, Ji Y, Pelleymounter LL, Eckloff BW, Wieben ED, Ames MM, Weinshilboum RM. Gemcitabine pharmacogenomics: cytidine deaminase and deoxycytidylate deaminase gene resequencing and functional genomics. Clin Cancer Res 2006;12:1794-803.

23) Giovannetti E, Del Tacca M, Mey V, Funel N, Nannizzi S, Ricci S, Orlandini C, Boggi U, Campani D, Del Chiaro M, Iannopollo M, Bevilacqua G, Mosca F, Danesi R. Transcription analysis of human equilibrative nucleoside transporter- 1 predicts survival in pancreas cancer patients treated with gemcitabine. Cancer Res 2006;66:3928-35.

24. Tibaldi C, Giovannetti E, Vasile E, Mey V, Laan AC, Nannizzi S, Di Marsico R, Antonuzzo A, Orlandini C, Ricciardi S, Del Tacca M, Peters GJ, Falcone A, Danesi R. Correlation of CDA, ERCC1, and XPD polymorphisms with response and survival in gemcitabine/cisplatin-treated advanced non-small cell lung cancer patients. Clin Cancer Res 2008;14:1797-803.

25. Pasqualetti G, Danesi R, Del Tacca M, Bocci G. Vascular endothelial growth factor pharmacogenetics: a new perspective for anti-angiogenic therapy. Pharmacogenomics 2007;8:4966. 
26. Lourenço GJ, Silva PM, Cardoso-Filho C, Schenka AA, Sagarra AF, Gurgel MS, Lima CS. The angiogenesis-related polymorphisms' role in breast cancer. J Clin Oncol 26:2008 (May 20 suppl; abstr 22027).

27. Jacobs EJ, Hsing AW, Bain EB, Stevens VL, Wang Y, Chen J, Chanock SJ, Zheng SL, Xu J, Thun MJ, Calle EE, Rodriguez C. Polymorphisms in angiogenesis-related genes and prostate cancer. Cancer Epidemiol Biomarkers Prev 2008;17:972-7.

28. Hsiao PJ, Lu MY, Chiang FY, Shin SJ, Tai YD, Juo SH. Vascular endothelial growth factor gene polymorphisms in thyroid cancer. J Endocrinol 2007;195:265-70.

29. García-Closas M, Malats N, Real FX, Yeager M, Welch R, Silverman D, Kogevinas M, Dosemeci M, Figueroa J, Chatterjee N, Tardón A, Serra C, Carrato A, García-Closas R, MurtaNascimento C, Rothman N, Chanock SJ. Large-scale evaluation of candidate genes identifies associations between VEGF polymorphisms and bladder cancer risk. PLoS Genet 2007;3:e29.

30. Försti A, Jin Q, Altieri A, Johansson R, Wagner K, Enquist K, Grzybowska E, Pamula J, Pekala W, Hallmans G, Lenner P, Hemminki K. Polymorphisms in the KDR and POSTN genes: association with breast cancer susceptibility and prognosis. Breast Cancer Res Treat 2007;101:8393. 